\title{
SECONDARY ELECTRON EMISSION MEASUREMENTS FOR TIN COATING ON THE STAINLESS STEEL OF SNS ACCUMULATOR RING VACUUM CHAMBER*
}

\author{
P. He ${ }^{\dagger}$, H.C. Hseuh, R. Todd \\ Collider-Accelerator Department, BNL, Upton, NY 11973, USA \\ B.Henrist, N. Hilleret, \\ LHC/VAC, CERN, Geneva, Switzerland \\ F.Le Pimpec, R.E. Kirby, M. Pivi, \\ SLAC, Stanford, CA 94309, USA \\ S. Kato, M. Nishiwaki, \\ KEK, Oho, Tsukuba, 305-0801, Japan
}

\begin{abstract}
BNL is responsible for the design and construction of the US Spallation Neutron Source (SNS) accumulator ring. Titanium Nitride (TiN) coating on the stainless steel vacuum chamber of the SNS accumulator ring is needed to reduce the secondary electron yield (SEY) and the undesirable resonant multiplication of electrons. The total SEY of TiN coated stainless steel material has been measured after coating samples were exposed to air and after electron and ion bombardment. We report here about TiN coating system setup at BNL and SEY measurements results at CERN, SLAC and KEK. We also present some simulation results of SNS accumulator ring electron-cloud effects using different SEY values.
\end{abstract}

\section{INTRODUCTION}

US Spallation Neutron Source (SNS) is an acceleratorbased neutron source being built in Oak Ridge, TN. The SNS is a partnership among six DOE national laboratories (Argonne, Brookhaven, Jefferson, Lawrence Berkeley, Los Alamos, and Oak Ridge). At a total cost of $\$ 1.4$ billion, construction began in 1999 and is scheduled for completion in 2006. Brookhaven is undertaking the design and construction of the SNS accumulator ring and beam transport lines. The operating pressure of the ring vacuum system [1] will be $10^{-9}$ Torr and is needed to minimize the beam-residual gas ionization. At the designed vacuum level, the rate of ionization and electron production is approximately $10^{-3}$ electrons per proton over the one-millisecond accumulation cycle. The electron cloud effect (ECE), due to multipacting, can limit the achievable proton intensity when excessive electrons are generated and trapped. The electron cloud density depends on characteristics of the proton beam (bunch length, charge and spacing) and the secondary electron yield and secondary emission spectrum of the vacuum chamber wall surface. The number of multipacting electrons can be effectively reduced by surface treatment of the vacuum pipe. The material of SNS ring vacuum chamber is stainless steel with a secondary emission yield (SEY) peak value typically in the range 2.5 3.0. A SEYlowering coating (TiN) has been applied to the SNS stainless steel vacuum chamber wall. The thickness of the TiN coating, typically about $100 \mathrm{~nm}$, is chosen because such coated section in PSR was shown to effectively suppress the electron flux by a factor of more than 100[2]. For critical elements, e.g., the injection kicker ceramic chambers and the ferrite of the extraction kicker inside the vacuum pipe, different pattern and thickness of the coating are selected to reduce the coupling impedance and to avoid eddy-current heating.

Magnetron DC sputtering was developed for SNS ring vacuum chamber TiN coating. The total secondary electron emission yield of TiN-coated stainless steel has been measured after samples of beam chamber material were exposed to air and again after electron and ion beam bombardment, as a function of the incident electron beam energy. The measurement values were used to simulate the electron build-up in the SNS straight section.

\section{TiN COATING PROCESSING}

Figure 1 shows the sputtering coating system for SNS chambers. The system has a water-cooled coaxial magnetron sputter source of $38 \mathrm{~mm}$ in diameter. The maximum transverse component of the magnetic field at the face of the titanium target is about 1000 Gauss. A DC power supply of $10 \mathrm{~kW}$ maximum is used as the power source. High purity argon and nitrogen are used as the sputtering and reactive gases, respectively.

All coatings have been done with the chamber at a temperature of $250^{\circ} \mathrm{C}$. The chamber pressure is usually reduced to $5 \times 10^{-7}$ Torr before deposition. At the beginning, the $\mathrm{Ti}$ cathode was pre-sputtered in a pure argon atmosphere for about $10 \mathrm{mins}$, and then the nitrogen is introduced into the chamber through a $6 \mathrm{~mm}$ diameter $\mathrm{Ti}$

*Work performed under the auspices of the U.S. Department of Energy

* SNS is managed by UT-Battelle, LLC, under contract DE-AC05-00OR22725 for the U.S. Department of Energy. SNS is a partnership of six national laboratories: Argonne, Brookhaven, Jefferson, Lawrence Berkeley, Los Alamos, and Oak Ridge.

†Email: phe@bnl.gov 
tube which runs the length of the cathode. Small holes (size gradually increased) located every $15 \mathrm{~cm}$ on the $\mathrm{Ti}$ tube provide even distribution of reactive gas, which is

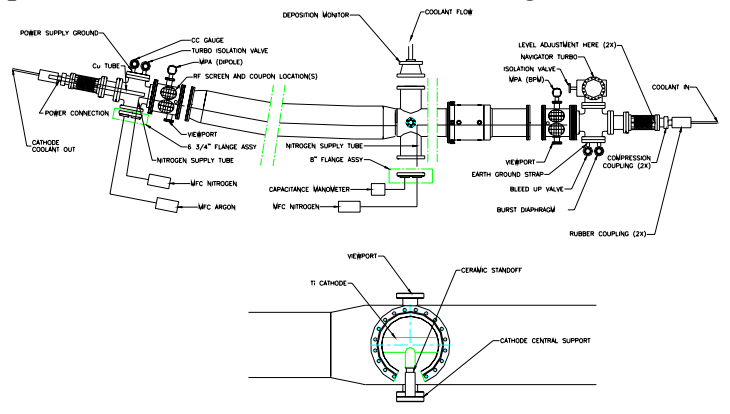

Figure 1. Schematics of magnetron sputtering coating setup for SNS ring halfcell chambers

necessary for obtaining a uniform longitudinal coating. Due to the pre-sputtering process, the sandwich (SS/Ti/TiN) coating cross-section was shaped, this sandwich layer will help to control the residual stress produced by the deposition process and improve the adhesion of the coating. The property of the coated films depends on the sputtering condition. Two process pressures were used during the sputtering, as listed in Table 1.

Table 1: Deposition Conditions for SNS Ring Vacuum Chambers

\begin{tabular}{lcc}
\hline & $\begin{array}{c}\text { High Pressure } \\
\text { (HP) Coating }\end{array}$ & $\begin{array}{c}\text { Low Pressure } \\
\text { (LP) Coating }\end{array}$ \\
\hline Total Pressure (mTorr) & 5.0 & 1.5 \\
Cathode Potential(V) & 300 & 350 \\
Cathode Current(A) & 10 & 10 \\
Argon Gas Flow(sccm) & 15.5 & 5.2 \\
Nitrogen Gas Flow $(\mathrm{sccm})$ & $4.1,1.6$ & $4.6,2.3$ \\
Chamber Temperature $\left({ }^{\circ} \mathrm{C}\right)$ & $200 \sim 250$ & $200 \sim 250$ \\
Film Thickness(nm) & $200 \sim 400$ & $200 \sim 400$ \\
\hline
\end{tabular}

\section{SECONDARY ELECTRON YIELD (SEY) MEASUREMENTS RESULTS}

Stainless steel witness coupons are put in the bottom of the spool piece at the both sides of the vacuum chamber (Figure 1). After the chambers were coated, the coupons were sent to CERN, SLAC and KEK for SEY measurements. The secondary electron yield depends on material, the primary electron energy, incident angle and surface state (the surface composition, the morphology the structure, and the roughness). The system used to measure SEY is composed of two coupled stainless steel UHV chambers where the pressure is in the low $10^{-10}$ Torr range in the measurement chamber and $10^{-9}$ Torr range in the "load lock" chamber. The measurement principle consists in recording simultaneously the current at the sample and a secondary collector (cage or Faraday cup). A very important feature for the measurement of the secondary electron yield is to control careful the electron dose needed for a measurement (typically a few nanoamps for the incident electron beam current). Ion gun is also installed in the measurement chamber, it was used for the sputtering cleaning. The $\mathrm{X}$-ray source is used to excite photoelectrons for XPS (X-ray Photoelectron Spectroscopy) analysis. The KEK SEY measurement setup is shown in Figure 2.

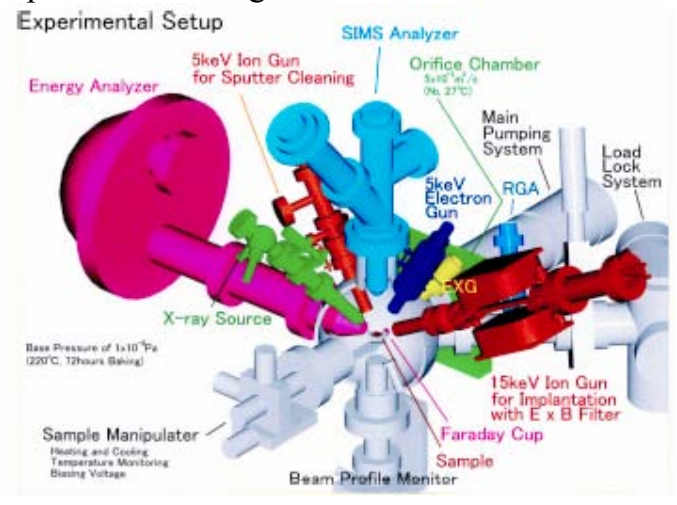

Figure 2. KEK SEY measurement experimental setup

\section{SEY vs coating process pressure}

The coated samples were measured in as received condition without in-situ bake. The difference between high pressure coating (5mTorr) and low pressure coating (1.5mTorr) is illustrated in Figure 3. From the results, we can see the samples coated at high pressure have lower SEY values (1.5 1.6) comparing to the samples coated at low pressure (SEY 2.0 2.7). The SEM (Scanning Electron Microscope) images reveal that coating at $5 \mathrm{mTorr}$ has rougher surface than the coating at $1.5 \mathrm{mTorr}[3]$. Similar surface roughness effect on SEY was also reported by CERN[4].

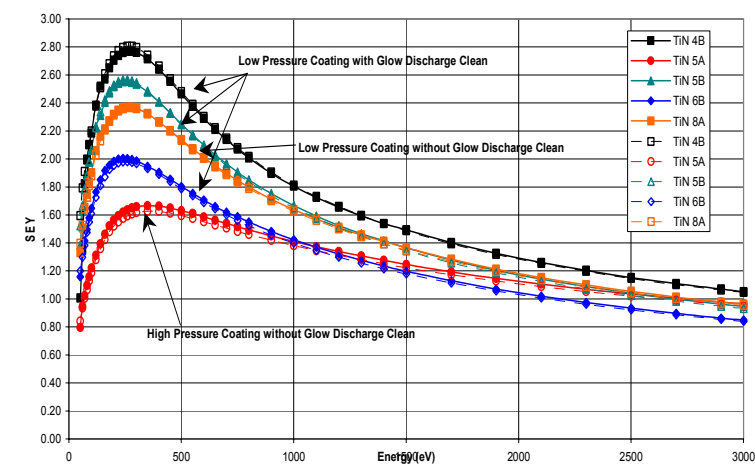

Figure 3. SEY measurement results of coating done at different process pressure

\section{SEY vs primary incident angle}

Angular SEY measurements were made on TiNcoated stainless steel coupons. Alignment of the electron beam incidence was made at normal and $60^{\circ}$ grazing angle. The angular dependence is shown in Figure 4.

\section{SEY vs conditioning}

The as-deposited/air-exposed SEY (e.g. TiN-coated stainless steel) is mainly determined by the presence of a surface layer of water and hydrocarbon[5]. Electron bombardment of this layer will serve to reduce the peak 
SEY close to the in-situ as-deposited TiN value(before air-exposed). The ion sputter-removal of surface gas is much more efficient than photon/electron bombardment. The SEY of TiN-SS sample was measured after electron dosing of $1.0 \times 10^{20}$ electrons- $\mathrm{cm}^{2}$ and Argon ion sputtering cleaning, the results are shown in Figure 5. XPS was used to measure the surface composition of the different conditions. Table 2 gives the atomic percentage of the surface composition after electron and ion conditioning.

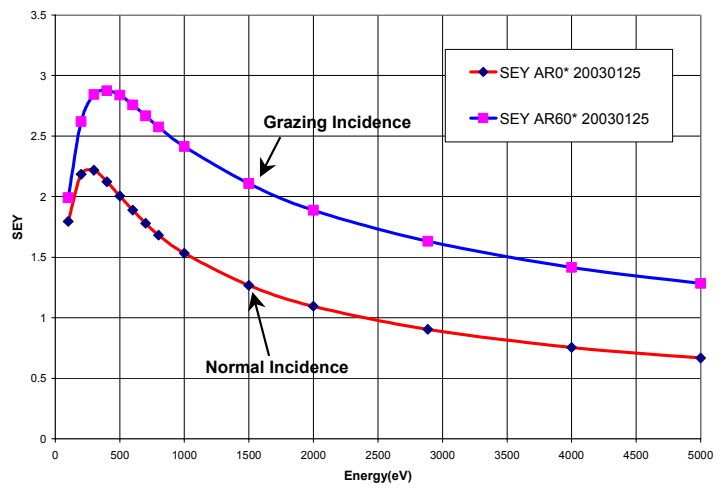

Figure 4. Angular SEY measurement

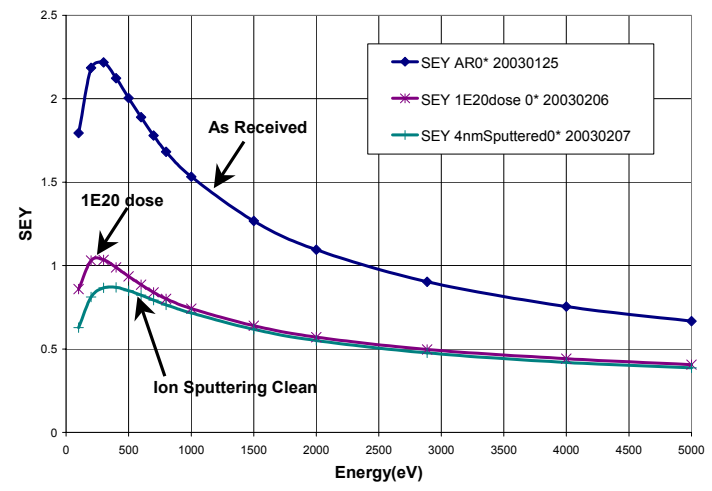

Figure 5. SEY before and after conditioning

Table 2. Atomic composition with XPS(at\%)

\begin{tabular}{|l|cccc|c|}
\hline & $\mathrm{Ti}$ & $\mathrm{N}$ & $\mathrm{C}$ & 0 & $\mathrm{SEY}$ (peak) \\
\hline As Received & 11 & 19 & 52 & 18 & 2.2 \\
1E20 electron dose & 15 & 25 & 45 & 15 & 1.04 \\
lon sputtering clean(4nm) & 47 & 53 & 0 & 0 & 0.87 \\
\hline
\end{tabular}

\section{SIMULATION USING MEASURED SEY DATA OF TIN/SS COATING}

The measured SEY values can be used to model the SNS electron cloud build-up by LBL code (POSINST)[6]. Important to such model is the behavior of the SEY as a function of both primary electron energy and incidence angle. This simulation study will improve the understanding of the dynamics of beam under the effects of the electron cloud in the SNS accumulator ring. The SNS machine parameters used for the simulation was shown in Table 3 . The electron buildup time line and the electron line density vs SEY are as shown in Figure 6 and Figure 7.

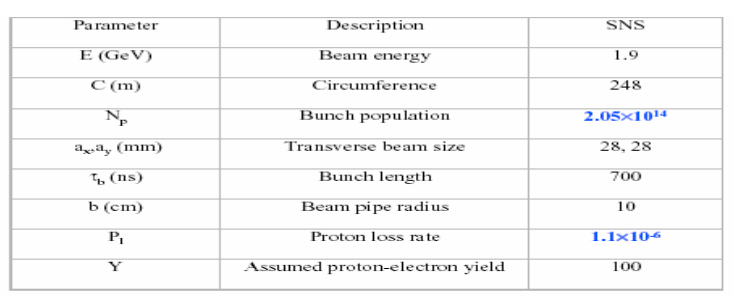

Table 3. SNS machine parameters using by simulation code

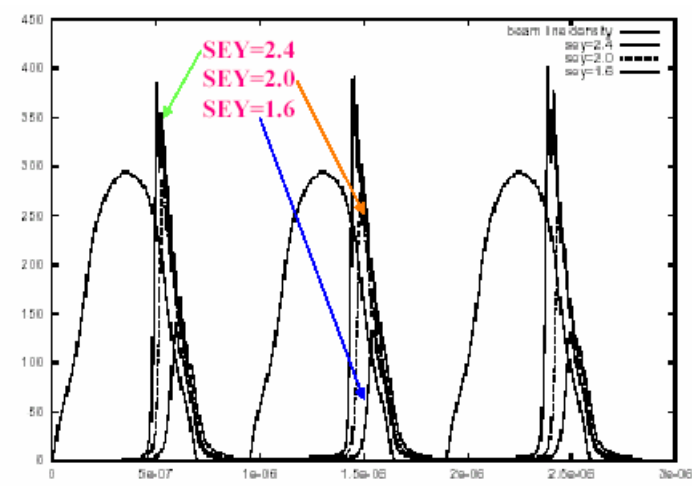

Figure 6. Electron build-up time line with different SEY

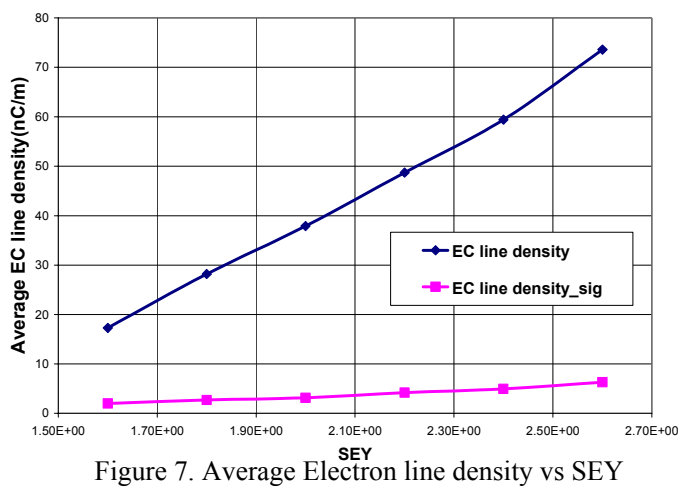

SUMMARY

We have investigated the SEY of the TiN coated on the stainless steel at different condition. Using the measurement results, the SNS accumulator ring electron cloud build-up is also simulated.

\section{ACKNOWLEDGEMENT}

The authors would like to thank Dr. M. Furman (LBNL) in providing the POSINST simulation code.

\section{REFERENCES}

[1] H.C. Hseuh, C.J. Liaw and M. Mapes, Proc. of 1999 Particle Accel. Conf., New York, pp1345-7 (2000).

[2] R. Macek, Proc. of ECLOUD'02 workshop, CERN (2002).

[3] P.He, et. al., Proc. of 2003 Particle Accel. Conf., Portland, OR, p788(2003).

[4] V.Baglin, et. al., Proc. of $7^{\text {th }}$ European Particle Accel. Conf., Vienna, Austria, pp217-221 (2000).

[5] J. Halbritter, J. de Physique 45, C2(1984)

[6] M.A. Furman and M. Pivi, Phys. Rev. ST Accel. Beams 5, 124404(2002). 